\title{
Technique of Optical Target Fusion Recognition Based On Fuzzy Evidence Theory
}

\author{
CHEN Jian-biao ${ }^{1, a}$, SUN Hua-yan ${ }^{2}$ and ZHENG Yong-hui ${ }^{1}$ \\ ${ }^{1}$ Company of Postgraduate Management, Academy of Equipment, Beijing 101416,China \\ ${ }^{2}$ Department of Photoelectric Equipment, Academy of Equipment, Beijing 101416,China \\ ageneral_cheng2041@163.com
}

Keywords: Information Fusion; Target Recognition; Fuzzy Evidence Theory; Membership Function

\begin{abstract}
As a technique of uncertainty inferring, D-S evidence theory is widely used in the region of information fusion. The problem is how to create the basic probability assignment function (BPAF) of evidence. A technique based on the membership function that can generate BPA is given. It is used to recognize optical target by extracting the peak power, pulse-broaden and flicker variance of the echo of active laser detection. The preponderances of non-imaging laser detection are high-precision, high-flexibility, and anti-electromagnetic interference, and that the cat's eye optical target is easy to be detected. Consequently, this paper presents the active probing of cat's eye target as an example. Firstly, the BPA of evidence is got by fuzzy theory. Then the result is got through combining them together by evidence theory. The experiment indicates that the technique can discriminate the cat's eye target exactly and improve the efficiency of recognition compared to identification with single attribute.
\end{abstract}

\section{Introduction}

D-S theory is a mathematical theory established by Dempster and Shafe in the end of 1960s and the beginning of 1970s, and generally this theory is used in the decision-level information fusion. It is an extension of the probability theory and is used to calculate the uncertain problems, fitting for expert systems, artificial intelligence and region of system decision [1,2] etc. In D-S evidence theory, the probability meaning uncertainty concept can be assigned to the event that is unknown. The frame of discrimination can indicate the uncertainty of a certain system or device. BPA is the carrier of evidence uncertainty. It is a key step to determine the support grade of proposition after getting the evidence. The value of BPA can signify the inference result of the degree of credibility about the target assumption. This judgment is affected by various factors, such as the environment around the target, the type of the sensors and so on. Many researches have been made to get the BPA with methods of neural network and the clustering of mean value of fuzzy $C[3,4,5,6]$ etc.This paper presents a method to get the BPA with fuzzy theory, and then it is applied to fuse the multi-attribute of the echo of cat's eye target with D-S theory.

\section{Basic Concepts}

Evidence Theory. The frame of discrimination is $\Theta=\left\{H_{1}, H_{2}, \ldots, H_{n}\right\}$. Its sub-propositions are mutually exclusive and can be counted. Every one of the proposition set can be assigned a basic probability assignment value $m\left(H_{i}\right)$ that indicates the credibility of the judgment of evidence. The representation $m: 2^{\Theta} \rightarrow[0,1]$ is called mass function, and that it also can be called basic probability assignment function (BPAF), it meets [7]: $m(\Phi)=0, \sum m(A)=1, A \subset \Theta, 2^{\Theta}$ is called the power set of the frame of discrimination, it is the number of all subsets of $\Theta$. And $\Phi$ is null set. A key step of D-S theory is to combine evidences. Evidence combination is a process of information fusion. Assuming $U$ and $V$ are two propositions of $\Theta$, and two sensors are used here, then: 


$$
m(A)= \begin{cases}\frac{1}{K} \sum_{U \cap V=A} m_{1}(U) m_{2}(V), & (A \neq \Phi) \\ 0, & (A=\Phi)\end{cases}
$$

is still an evidence. This expression is named as Dempster-Shafe formula. The parameter $K$ is to normalize the combined evidence. When the conflict exists between two evidences, namely $K$ approximating to 1 , the section of conflicting should be set 0 , and the rest of mass are normalized by $K^{-1}$. Some improved methods about rule of evidence combination are researched $[8,9]$.

$$
K=1-\sum_{U \cap V=\Phi} m_{1}(U) m_{2}(V)=\sum_{U \cap V \neq \Phi} m_{1}(U) m_{2}(V)>0
$$

Fuzzy Theory. Boundary fuzzy is the basic difference between fuzzy set and classical set, which means that an element can belong to a fuzzy set or not. For a domain of discourse area $U$, the any mapping of the closed interval from 0 to 1 could determine a fuzzy subset, the $u_{T}(\mathrm{x})$ is called the membership function of the fuzzy subset $T$. The value indicates the degree of the membership of $x$ belonging to $T$. The closer it is to 1 , the degree of membership is higher and vice versa. If there are two kinds of targets, namely $A$ and $B$, the membership functions of which are $\mu_{A}$ and $\mu_{B}$ separately, then the unknown probability of target is: $\Phi=1-\max \left(\mu_{A}, \mu_{B}\right)$. After normalizing, the BPA of a certain attribute can be gotten form Eq. 4, $i$ is the index of a certain sensor or attribute of target, and $\mathrm{A}$ and $\mathrm{B}$ are the type of targets. The normalization guarantees that the summation of BPA is 1 . Then $\mathrm{D}-\mathrm{S}$ evidence theory can be used to combine the evidence in the process of decision-level fusion.

$$
m_{i}(\mathrm{~A})=\frac{\mu_{i}(\mathrm{~A})}{\mu_{i}(\mathrm{~A})+\mu_{i}(\mathrm{~B})+\mu_{i}(\Phi)}, m_{i}(\mathrm{~B})=\frac{\mu_{i}(\mathrm{~B})}{\mu_{i}(\mathrm{~A})+\mu_{i}(\mathrm{~B})+\mu_{i}(\Phi)}, m_{i}(\Phi)=\frac{\mu_{i}(\Phi)}{\mu_{i}(\mathrm{~A})+\mu_{i}(\mathrm{~B})+\mu_{i}(\Phi)}
$$

Triangle function, index function, and Gauss function are the common forms of membership function, it depends on the real condition to determine that which kind of function will be used. The triangle function is applied here to show the ambiguity of the target's attribute. A triangle function can be expressed as $(a, b, c)$. The expression of the triangle membership function [10] is as follow referring to Eq. 4:

$$
\mu_{A}(\mathrm{x})\left\{\begin{array}{l}
0, x<a \\
\frac{x-a}{b-a}, a \leq x \leq b \\
\frac{c-x}{c-b}, \mathrm{~b} \leq x \leq c \\
0, x>c
\end{array} .\right.
$$

\section{Experiment and analysis}

In the case that the system observes an optical cat's eye target [11] , many attribute values that are helpful to target recognition can be gotten by processing and analyzing the echo signal, such as peak power, pulse-broaden, flicker variance and so on. The three attributes of echo indicate different features of the optical target. For example, the peak power shows the reflectivity of the target. Moreover, the pulse-broaden can express the depth of distance of the target. The flow chart of the target recognition using fuzzy evidence theory is as follow referring to Fig. 1: 


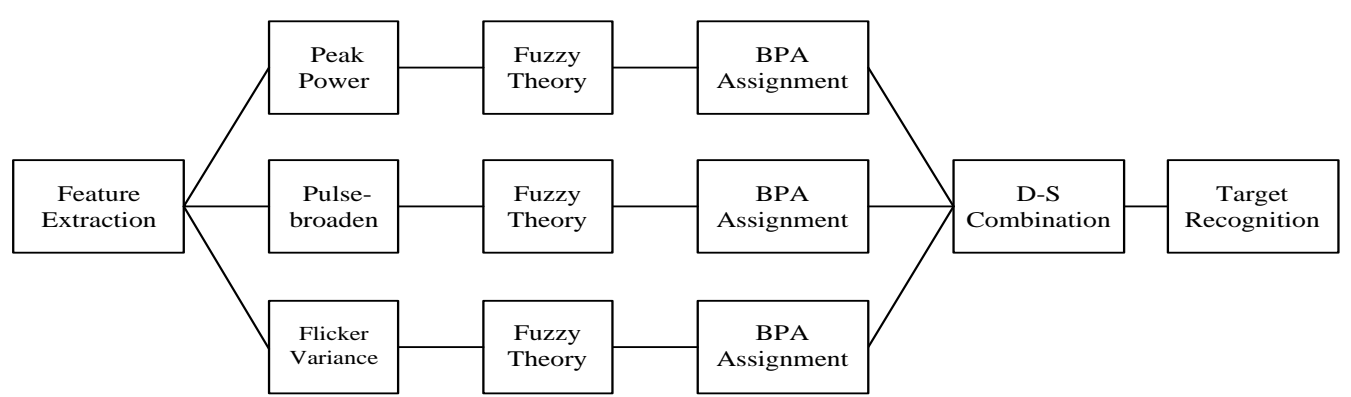

Fig. 1. The flow chart of the information fusion of target recognition

In this experiment, the sensor was a laser detector aiming at wave length of $1064 \mathrm{~nm}$, and the target was a cat's eye target and the background was supposed as the diffused reflector. The three attributes mentioned before were used to judge the type of the target. The purpose of this test is to estimate if the object is a cat's eye target or not. For getting the extent of matching of every attribute, the membership function of Eq. 5 was used:

$$
u(\mathrm{z})= \begin{cases}1-\frac{|z-u|}{2 \sigma}, & |z-u|<2 \sigma \\ 0, & |z-u|>2 \sigma\end{cases}
$$

In this formula, $z$ is the value of measurement, $(\mu, \sigma)$ are the mean value and variance. The measurement of attributes are showed in Table 1, here A1, A2, and A3 are on behalf of the peak power, pulse-broaden, and flicker variance separately.

Table 1 Parameter and value of measure

\begin{tabular}{|c|c|c|c|c|c|}
\hline \multirow{2}{*}{$\begin{array}{c}\text { Target } \\
\text { attribute }\end{array}$} & \multicolumn{2}{|c|}{ Cat's eye target } & \multicolumn{2}{|c|}{ Background } & \multirow{2}{*}{$\begin{array}{c}\text { Value of } \\
\text { measure }\end{array}$} \\
\cline { 2 - 5 } A1 & $\mu$ & $\sigma$ & $\mu$ & $\sigma$ & 15 \\
\hline A2 & $2000[\mathrm{nw}]$ & 200 & $200[\mathrm{nw}]$ & $1900[\mathrm{nw}]$ \\
\hline A3 & $3[\mathrm{~ns}]$ & 0.6 & $5[\mathrm{~ns}]$ & 2.3 & $3.3[\mathrm{~ns}]$ \\
\hline
\end{tabular}

Computing with Eq. 6, the extent of supporting for cat's eye optical target and background obtained from the attributes are in Table 2. After normalizing, the BPA of a certain attribute can be gotten form Eq. 3.The fusion result generated by the three target attributes are as follow in Table 3.

Table 2 Degree of supporting of the fuzzy proposition

\begin{tabular}{|c|c|c|}
\hline Target attribute & Cat's eye target $\left(\mu_{A}\right)$ & Background $\left(\mu_{B}\right)$ \\
\hline A1 & 0.75 & 0 \\
\hline A2 & 0.75 & 0.63 \\
\hline A3 & 0.6875 & 0.5 \\
\hline
\end{tabular}

According to Eq. 3, the BPA are as follow:

$$
\begin{gathered}
m_{A 1}(\mathrm{~A})=0.75, m_{A 1}(\mathrm{~B})=0, m_{A 1}(\Phi)=0.25 ; \\
m_{A 2}(\mathrm{~A})=0.46, m_{A 2}(\mathrm{~B})=0.386, m_{A 2}(\Phi)=0.154 ; \\
m_{A 3}(\mathrm{~A})=0.45, m_{A 3}(\mathrm{~B})=0.34, m_{A 3}(\Phi)=0.21 ;
\end{gathered}
$$

Table 3 Result of evidence theory fusion

\begin{tabular}{|c|c|c|c|}
\hline Attribute fusion & $m_{1}$ & $m_{1}, m_{2}$ & $m_{1}, m_{2}, m_{3}$ \\
\hline \multirow{3}{*}{ Fusion result } & $m(\mathrm{~A})=0.75$ & $m(\mathrm{~A})=0.8101$ & $m(\mathrm{~A})=0.8426$ \\
\cline { 2 - 4 } & $m(\mathrm{~B})=0$ & $m(\mathrm{~B})=0.1360$ & $m(\mathrm{~B})=0.1404$ \\
\cline { 2 - 4 } & $m(\Phi)=0.25$ & $m(\Phi)=0.0539$ & $m(\Phi)=0.0170$ \\
\hline
\end{tabular}

Form table 3, the maximal BPA of the evidence combination is $m(\mathrm{~A})=0.843$, it indicates that the target is more likely cat's eye target . Along with the accumulating of the mass function of evidence, 
the degree of supporting that the target is A is increasing. Meanwhile, $m(\Phi)$ is decreasing that means the reduction of the system uncertainty. The result shows that the result of calculation is matched with the experiment. We can make a summary:

1) Multi-attribute judgment is more efficient and exact than single attribute.

2) The method that combined fuzzy set theory with evidence decision is credible to some extent.

\section{Conclusion}

In this paper, fuzzy theory and evidence theory are combined to overcome the shortage during application of the D-S theory. A technique based on fuzzy set theory and the membership functions that can generate BPA is given. Then the BPA of evidence is combined with D-S theory to make decision. Moreover, the validity and efficiency of the method are tested and verified through experiment. The problem is that triangle membership function is not the optimal choice, the more accurate method is expected to be found. In the next step, combining fuzzy evidence theory with other relevant theory is a significant way to solve problems in decision-level information fusion.

\section{References}

[1] Lsurent Lecornu, Renaud Debon, Wojtek Komorniczak. Probabilistic and fuzzy information fusion applied to radar system ranking. Conference Proceedings of SPIE Multisensor, Multisource Information Fusion: Architetures, Algorhims, and Application. B. V. Dasarathy, Editor, vol. 5099,pp 123-132, 2003.

[2] Jaime Esteban, Andrew Starr, Robert Willetts etc. A review of data fusion models and architectures: towards engineering guidelines[J]. Neural Comput \& Applic,2005,14:273-281.

[3] LI Yun-bin, LI Hui, WANG Yun-fei etc. BPA generation method based on the similarity of fuzzy numbers[J]. Modern Electronics Technique,2011,34(15):5-7.

[4] Wang Feng-chao, Liu Xing-tang, Huang Shu-cai etc. Target fusion detection with multi-feature based on fuzzy evidence theory[J]. Acta Electronica Sinica,2010,30(3):713-719.

[5] LI Hui, PAN Kai,ZHANG Xin etc. Multi-sensor information fusion algorithm based on fuzzy theory. Computer Engineering and Applications,2012,48(10):128-131.

[6] HAN Feng, YANG Wan-hai, YUAN Xiao-guang etc. Evidence theory information fusion method based on fuzzy set[J]. Control and Decision,2010,25(3):449-452.

[7] Naga R. Mudigonda, Ray Kacelenga, Kacelenga, David Erickson. The application of Dempster-Shafe theory for landmine detection. Conference Proceedings of SPIE Multisensor, Multisource Information Fusion: Architetures, Algorhims, and Application. B. V. Dasarathy, Editor, vol. 5099,pp 103-112, 2003.

[8] Yager RR. On the Dempster-Shafer framework and new combination rules[J]. Information Science,1987,41(2):93-138.

[9] Catherine K Murphy. Combining belief functions when evidence conflicts[J]. Decision Support Systems,2000,29(1):1-9.

[10]Ding Hao, JI Hai-feng, Huang Zhi-yao, LI Hai-qing etc. Data fusion algorithm based on fuzzy logic. Proceedings of the 5th World Congress on Inte1ligent Control and Automation. Hangzhou, China, 2004:3101-3103.

[11]DONG Xiao-gang, ZHU Hai-jun, YIN Gang etc. Detection distance analysis of pulsed laser based on cat's eye effect[J]. Laser \& Infrared,2011,41(1):67-70. 